\title{
Tecnologias de Informação e Comunicação no ensino de Enfermagem durante a pandemia da COVID-19: Revisão integrativa
}

Education and Communication Technologies in Nursing teaching during the COVID-19 pandemic: Integrative Review

Tecnologías de Educación y Comunicación en la Enfermería durante la pandemia COVID-19: Revisión Integrativa

\section{Resumo}

Objetivo: conhecer as tecnologias educacionais e de comunicação utilizadas no ensino da enfermagem durante a pandemia da COVID-19, bem como refletir como cada tecnologia contribuiu no processo de ensino-aprendizagem nesse cenário. Metodologia: Trata-se de uma revisão integrativa de literatura. A busca eletrônica foi realizada no mês de fevereiro de 2021 nas bases de dados Biblioteca Virtual em Saúde, MEDLINE/PubMed, Cumulative Index to Nursing and Allied Health Literature e Web of Science com as palavras chaves selecionadas nos Descritores em Ciência da Saúde e Medical Subject Headings Resultados: Foram encontrados 80 estudos, após aplicação dos critérios de elegibilidade, restaram 8 artigos para elaboração da síntese. Neste estudo, identificamos o uso de diferentes tecnologias para o ensino da enfermagem, destacando-se a simulação clínica em ambiente virtual, plataformas digitais de ensino e plataformas de comunicação online. Conclusão: O uso destas tecnologias demonstra eficiência ao substituir os modelos tradicionais de aprendizagem durante a crise sanitária causada pelo novo coronavírus, entretanto evidenciaram-se dificuldades na incorporação destas ferramentas por alunos e professores, principalmente relacionadas a problemas de infraestrutura e capacitação tecnológica dos educadores.

Palavras-chave: Tecnologia educacional; COVID-19; Educação em enfermagem; Ensino.

\begin{abstract}
Objective: to know the educational and communication technologies used in nursing education during the COVID-19 pandemic, as well as to reflect how each technology contributed to the teaching-learning process in this scenario. Methodology: This is an integrative literature review. The electronic search was performed in the Virtual Health Library, MEDLINE / PubMed, Cumulative Index to Nursing and Allied Health Literature and Web of Science databases with the keywords selected in the Health Science Descriptors and Medical Subject Headings. Results: 80 studies were found, after applying the eligibility criteria, 8 articles remained for the synthesis. In this study, we identified the use of different technologies for teaching nursing, highlighting clinical simulation in a virtual environment, digital teaching platforms and online communication platforms. Conclusion: The use of these technologies proved to be efficient to replace traditional models of learning during the health crisis caused by the new coronavirus, however difficulties were evidenced in the incorporation of these tools by students and teachers, mainly related to problems of infrastructure and technological training of educators.
\end{abstract}

Keywords: Educational technology; COVID-19; Education nursing; Teaching.

\section{Resumen}

Objetivo: conocer las tecnologías educativas y de comunicación utilizadas en la educación de enfermería durante la pandemia de COVID-19, así como reflejar cómo cada tecnología contribuyó al proceso de enseñanza-aprendizaje en este escenario. Metodología: Se trata de una revisión integradora de la literatura. La búsqueda electrónica se realizó en 
las bases de datos Biblioteca Virtual en Salud, MEDLINE / PubMed, Cumulative Index to Nursing and Allied Health Literature y Web of Science con las palabras clave seleccionadas en los Descriptores de Ciencias de la Salud y Medical Subject Headings. Resultados: Se encontraron 80 estudios, luego de aplicar los criterios de elegibilidad, quedan 8 artículos para la síntesis. En este estudio, identificamos el uso de diferentes tecnologías para la enseñanza de la enfermería, destacando la simulación clínica en un entorno virtual, plataformas de enseñanza digital y plataformas de comunicación online. Conclusión: El uso de estas tecnologías demostró ser eficiente para reemplazar los modelos tradicionales de aprendizaje durante la crisis de salud provocada por el nuevo coronavirus, sin embargo se evidenciaron dificultades en la incorporación de estas herramientas por parte de estudiantes y docentes, principalmente relacionadas con problemas de infraestructura y tecnología. formación de educadores.

Palabras clave: Tecnología educativa; COVID-19; Educación en enfermería; Ensenãnza.

\section{Introdução}

A formação em saúde é influenciada por diversos fatores que compõem a sociedade, entre eles social, político, econômico e também as tendências de mercado profissional. Estes envolvem o sistema de saúde brasileiro e as políticas públicas para a formação de profissionais de saúde, que devem responder às necessidades da sociedade brasileira, tanto na produção de conhecimento como também na prestação de serviços (Góes, de Camargo, Hara \& Fonseca, 2014).

Com a pandemia do novo coronavírus declarada pela Organização Mundial de Saúde (OMS) em 11 de março de 2020 , medidas para manter o distanciamento social foram adotadas para reduzir a disseminação da doença (World Health Organization, 2020). No Brasil, dentre as medidas de contenção, o Ministério da Educação (MEC) decretou em 16 de março de 2020 a suspensão das aulas presenciais e o fechamento das instituições de ensino (Brasil, 2020a). Em 17 de março de 2020, o MEC instituiu a Portaria $\mathrm{n}^{\circ} 343$ que autorizou as instituições a substituírem as aulas presenciais por aulas em meios digitais, enquanto durar a pandemia do novo coronavírus (Brasil, 2020b).

Nesse contexto, os cursos de formação superior, incluindo os da área da saúde como Enfermagem, obrigaram-se a adotar métodos de ensino remoto para continuidade do ensino como medida de prevenção contra a Covid-19. O ensino remoto (ER) consiste na transmissão das aulas em tempo real, favorecendo a interação entre aluno e professor por meios digitais (Saldanha, 2020). O Ensino Remoto Emergencial (ERE), por sua vez, é um meio temporário de dar continuidade às aulas, no qual continuam os mesmos estudantes e professores do formato presencial, porém a intermediação se dá via digital, diante da impossibilidade de encontros presenciais (Lira, Adamy, Teixeira \& Silva, 2020).

Deve-se destacar, no entanto, que o ensino remoto diferencia-se do Ensino à Distância (EaD). O EaD, apesar de também utilizar meios digitais e tecnológicos, não permite interação entre estudantes e docentes, pois nessa modalidade de ensino os conteúdos são previamente preparados; as aulas não são síncronas, pois são gravadas; os materiais didáticos são padronizados e os tutores ficam disponíveis apenas para tirar dúvidas dos alunos (Lira et al, 2020).

No que se refere ao ensino e formação profissional da categoria, o Conselho Federal de Enfermagem (COFEN) considera que o EaD pode ser uma estratégia interessante para cursos de pós-graduação, formação continuada e aperfeiçoamentos. No entanto, a posição do conselho é contrária ao EaD em cursos técnicos e de graduação (Conselho Federal de Enfermagem, 2020).

O EaD em Enfermagem é autorizado pela portaria n 2.117 de dezembro de 2019 (Brasil, 2019), do MEC, a qual permite que os cursos de graduação presencial, incluindo os da área da saúde, ofereçam até 40\% da carga horária na modalidade EaD. A adoção dessa modalidade de ensino na enfermagem, no entanto, prejudica a interação entre professores e alunos em tempo real e no mesmo espaço, consequentemente impactando o desenvolvimento das competências e habilidades profissionais do enfermeiro ou técnico de enfermagem, as quais requerem o contato humano para o ato de cuidar (Teixeira, Fernandes, Andrade, Silva, Rocha \& Lima, 2013).

Tanto o EaD, introduzido no Brasil em 1996, quanto o ER, popularizado no contexto da pandemia da Covid-19, são modalidades de ensino que dependem do uso de tecnologias educacionais digitais (TED) e tecnologias da informação e 
comunicação (TICs) (Paese, 2012).

As TED vêm sendo amplamente adotadas nos cursos da área da saúde possibilitando maior flexibilidade e diversificação, de modo que o estudante acesse os conteúdos em tempo e local que desejar. Podem ser utilizados recursos como vídeos, games, hipertextos, ambientes virtuais, aplicativos, simulações online, entre outros recursos usados em atividades presenciais ou a distância (Lathi, Hatonen \& Valimaki, 2014). Apesar de muitas vezes as TED apresentarem alto custo, esses materiais digitais podem dinamizar e facilitar o aprendizado de procedimentos de enfermagem, rompendo com o ensino tradicional, que demonstra e repete procedimentos (Silveira \& Cogo, 2017).

Já as TIC podem ser compreendidas como recursos tecnológicos que, quando integrados, proporcionam a comunicação em diversos processos, inclusive o de ensino-aprendizagem. São tecnologias que reúnem, distribuem e compartilham informações, como: softwares, hardwares, redes ou celulares (Oliveira, 2015). O aumento e modernização das TICs e sua utilização nos espaços de ensino permite novas formas de trabalhar os conteúdos curriculares, além de proporcionar maior interação entre aluno e professor durante o processo de ensino (Andrade, 2019).

Um desafio encontrado no uso das TED e TIC é a falta de capacitação dos professores para o seu uso e a dificuldade de acesso dos alunos. Um estudo realizado com docentes de educação física obteve como relato que as principais dificuldades encontradas no ensino remoto foram adaptação às novas ferramentas tecnológicas e sobrecarga de trabalho (Godoi, Beraldo, Kawashima, Gomes \& Caneva, 2020).

Desse modo, é necessário que o professor elabore estratégias para a aplicação dessas novas tecnologias, de forma que essas contribuam no processo de ensino e aprendizagem e proporcionem ao aluno uma compreensão geral do conteúdo, utilizando a tecnologia como instrumento pedagógico (Oliveira, 2015).

Conhecer a capacidade que as TED e TIC têm para contribuir com a educação é fundamental para a promoção do conhecimento, considerando tanto o cenário de expansão do uso dessas tecnologias na formação em saúde, quanto o contexto atípico vivenciado pela sociedade em tempos de pandemia do novo coronavírus.

Neste contexto, o objetivo desta revisão é conhecer as tecnologias educacionais e de comunicação utilizadas no ensino da enfermagem durante a pandemia da Covid-19, bem como refletir como cada tecnologia contribuiu no processo de ensinoaprendizagem nesse cenário.

\section{Metodologia}

Este estudo trata de uma revisão integrativa de literatura, cujo a finalidade é reunir e sintetizar vários estudos publicados sobre determinado assunto permitindo chegar a conclusões finais, proporcionando aos profissionais, resultados relevantes que podem fundamentar suas condutas a partir do conhecimento crítico e científico (Mendes, Silveira \& Galvão, 2008).

O desenvolvimento desta revisão seguirá as orientações do The Preferred Reporting Systematic Reviews and MetaAnalyses of Studies - PRISMA (Moher, Liberati, Tetzlaff \& Altman, 2009). Este constitui-se de 6 etapas, conforme as instruções de Mendes et al (2008): 1) identificação da questão de pesquisa, 2) estabelecimento de critérios para inclusão e exclusão de estudos, 3) definição das informações a serem extraídas dos estudos selecionados,4) avaliação dos estudos incluídos na revisão integrativa, 5) interpretação dos resultados e 6) apresentação da revisão.

A pergunta norteadora da pesquisa foi elaborada através da estratégia do acrônimo PICo: População, Intervenção, Contexto (Peters, Marnie, Tricco, Pollock, Munn, Alexander \& Khalil, 2020). Sendo P: ensino remoto emergencial em enfermagem, I: tecnologias educacionais e de comunicação, C: pandemia da Covid-19. A partir disso foi estabelecida a seguinte questão de pesquisa: Quais são as tecnologias educacionais e de comunicação utilizadas no ensino de enfermagem durante a pandemia da Covid-19? 
A busca eletrônica foi realizada no mês de fevereiro de 2021 nas bases de dados Biblioteca Virtual em Saúde (BVS), MEDLINE/PubMed, Cumulative Index to Nursing and Allied Health Literature (CINAHL) e Web of Science (WOS) com as palavras chaves selecionadas nos Descritores em Ciência da Saúde (DeCS) e Medical Subject Headings (MeSH). Seguindo uma estratégia de busca elaborada por um bibliotecário, as palavras centrais utilizadas foram "Tecnologia Educacional", "COVID-19" e "Educação em Enfermagem" e demais palavras derivadas destas, nos idiomas português, inglês e espanhol, como se pode observar no quadro abaixo (Quadro 1).

Quadro 1 - Estratégia Utilizada para captação de artigos na base de dados.

\begin{tabular}{|c|}
\hline Estratégia utilizada para a busca dos estudos \\
\hline 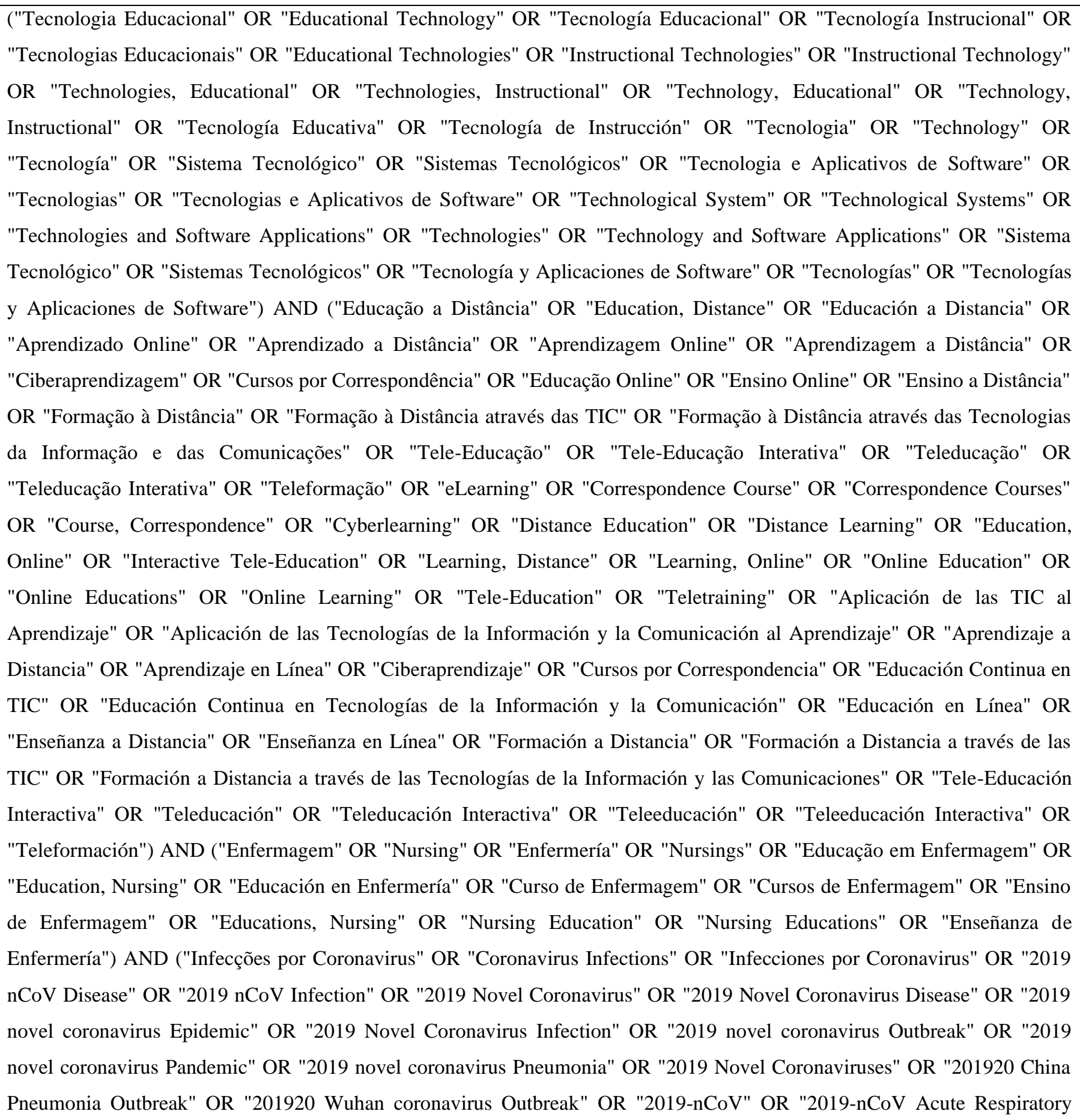 \\
\hline
\end{tabular}


Disease" OR "2019-nCoV Disease" OR "2019-nCoV Diseases" OR "2019-nCoV Epidemic" OR "2019-nCoV Infection" OR "2019-nCoV Infections" OR "2019-nCoV Outbreak" OR "2019-nCoV Pandemic" OR "2019-nCoV Pneumonia" OR "2019new coronavirus Epidemic" OR "2019-novel coronavirus (2019-nCoV) Infection" OR "2019-novel coronavirus Pneumonia" OR "Brote de Neumonía de China de 2019-2020" OR "Brote por 2019-nCoV" OR "Brote por el Coronavirus 2019-nCoV" OR "Brote por el coronavirus de Wuhan" OR "Brote por el Coronavirus de Wuhan de 2019-2020" OR "Brote por el Nuevo Coronavirus (2019-nCoV)" OR "Brote por el Nuevo Coronavirus 2019" OR "Coronavirus 2, SARS" OR "Coronavirus Disease 19" OR "Coronavirus Disease 2019" OR "Coronavirus Disease 2019 Virus" OR "Coronavirus Disease-19" OR "Coronavirus Infection" OR "Coronavirus, 2019 Novel" OR "Coronavirus, Wuhan" OR "COVID 19" OR "COVID 19 Pandemic" OR "COVID 19 Virus" OR "COVID 19 Virus Disease" OR "COVID 19 Virus Infection" OR "COVID19" OR "COVID-19" OR "COVID-19 Pandemic" OR "COVID-19 Pandemics" OR "COVID-19 Virus" OR "COVID-19 Virus Disease" OR "COVID-19 Virus Diseases" OR "COVID-19 Virus Infection" OR "COVID-19 Virus Infections" OR "COVID-19 Viruses" OR "COVID19*" OR "COVID-19*" OR "COVID-2019*" OR "Disease 2019, Coronavirus" OR "Disease, 2019-nCoV" OR "Disease, COVID-19 Virus" OR "Doença pelo Novo Coronavírus (2019-nCoV)" OR "Doença por Coronavírus 2019-nCoV" OR "Doença por Novo Coronavírus (2019-nCoV)" OR "Enfermedad por Coronavirus 2019-nCoV" OR "Enfermedad por el Nuevo Coronavirus (2019-nCoV)" OR "Epidemia pelo Coronavírus de Wuhan" OR "Epidemia pelo Coronavírus em Wuhan" OR "Epidemia pelo Novo Coronavírus (2019-nCoV)" OR "Epidemia pelo Novo Coronavírus 2019" OR "Epidemia por 2019nCoV" OR "Epidemia por Coronavírus de Wuhan" OR "Epidemia por Coronavírus em Wuhan" OR "Epidemia por el Coronavirus de Wuhan" OR "Epidemia por el Nuevo Coronavirus (2019-nCoV)" OR "Epidemia por el Nuevo Coronavirus 2019" OR "Epidemia por Novo Coronavírus (2019-nCoV)" OR "Epidemia por Novo Coronavírus 2019" OR "Novel Coronavirus Pneumonia" OR "Novel Coronavirus, 2019" OR "Pandemia por el Coronavirus de Wuhan" OR "Pandemia por el Nuevo Coronavirus (2019-nCoV)" OR "Pandemia por el Nuevo Coronavirus 2019" OR "Pandemic, COVID-19" OR "SARS Coronavirus 2" OR "SARS Coronavirus 2 Infection" OR "SARS CoV 2 Infection" OR "SARS CoV 2 Virus" OR "SARS-CoV19" OR "SARS-CoV-2" OR "SARS-CoV-2 Infection" OR "SARS-CoV-2 Infections" OR "SARS-CoV-2 Virus" OR "SARSCoV-2 Viruses" OR "SARS-CoV-2019" OR "Severe Acute Respiratory Syndrome Coronavirus 2" OR "Surto de Coronavírus de Wuhan" OR "Surto de Pneumonia da China 2019-2020" OR "Surto de Pneumonia na China 2019-2020" OR "Surto pelo Coronavírus 2019-nCoV" OR "Surto pelo Coronavírus de Wuhan" OR "Surto pelo Coronavírus de Wuhan de 2019-2020" OR "Surto pelo Novo Coronavírus (2019-nCoV)" OR "Surto pelo Novo Coronavírus 2019" OR "Surto por 2019-nCoV" OR "Surto por Coronavírus 2019-nCoV" OR "Surto por Coronavírus de Wuhan" OR "Surto por Coronavírus de Wuhan de 2019-2020" OR "Surto por Novo Coronavírus (2019-nCoV)" OR "Surto por Novo Coronavírus 2019" OR "Virus Disease, COVID-19" OR "Virus Infection, COVID-19" OR "Virus, COVID-19" OR "Virus, SARS-CoV-2" OR "Wuhan Coronavirus" OR "Wuhan coronavirus Epidemic" OR "Wuhan coronavirus Infection" OR "Wuhan coronavirus Outbreak" OR "Wuhan coronavirus Pandemic" OR "Wuhan coronavirus Pneumonia" OR "Wuhan Seafood Market Pneumonia" OR "Wuhan Seafood Market Pneumonia Virus")

Fonte: Autores (2021).

Os critérios de inclusão adotados na revisão foram: artigos publicados de março de 2020 a janeiro de 2021, nos idiomas português, inglês ou espanhol, com textos completos e gratuitos, tendo como público alvo estudantes de enfermagem, e possuir como intervenção alguma tecnologia educacional e/ou de comunicação. E os critérios de exclusão foram: artigos duplicados, estudo de revisão, editoriais e a ausência de informação relativa ao objetivo da pesquisa.

A seleção dos artigos foi realizada por dois revisores independentes, caso houvesse discordância, era chamado um terceiro revisor para análise final conforme as instruções do Instituto Joanna Briggs (Peters et al, 2020). Os resultados da 
pesquisa foram exportados para a ferramenta State of the Art through Systematic Review (StArt ${ }^{\circledR}$ ), e passaram por um processo de filtragem, sendo removidos os textos duplicados.

Foram extraídas informações como: autores do artigo; ano de publicação; país onde foi desenvolvido; objetivos, tipo de estudo, tecnologia contemplada e principais resultados (Peters et al, 2020). Por se tratar de uma revisão integrativa de literatura, o projeto não necessitou de aprovação de Comissão de Ética em pesquisa conforme Resolução no 466 de 2012 do Conselho Nacional de Saúde/Comissão Nacional de Ética em Pesquisa.

\section{Resultados}

Foram encontrados quatro artigos no portal da Web of science, 10 na PubMed, 14 na CINAHL, 14 na SCOPUS e 38 na BVS, totalizando 80 achados. O fluxograma abaixo sintetiza o processo de captação, seleção e inclusão dos estudos desta revisão integrativa (Figura 1).

Figura 1 - Fluxograma prisma para captação, seleção e inclusão de estudos.
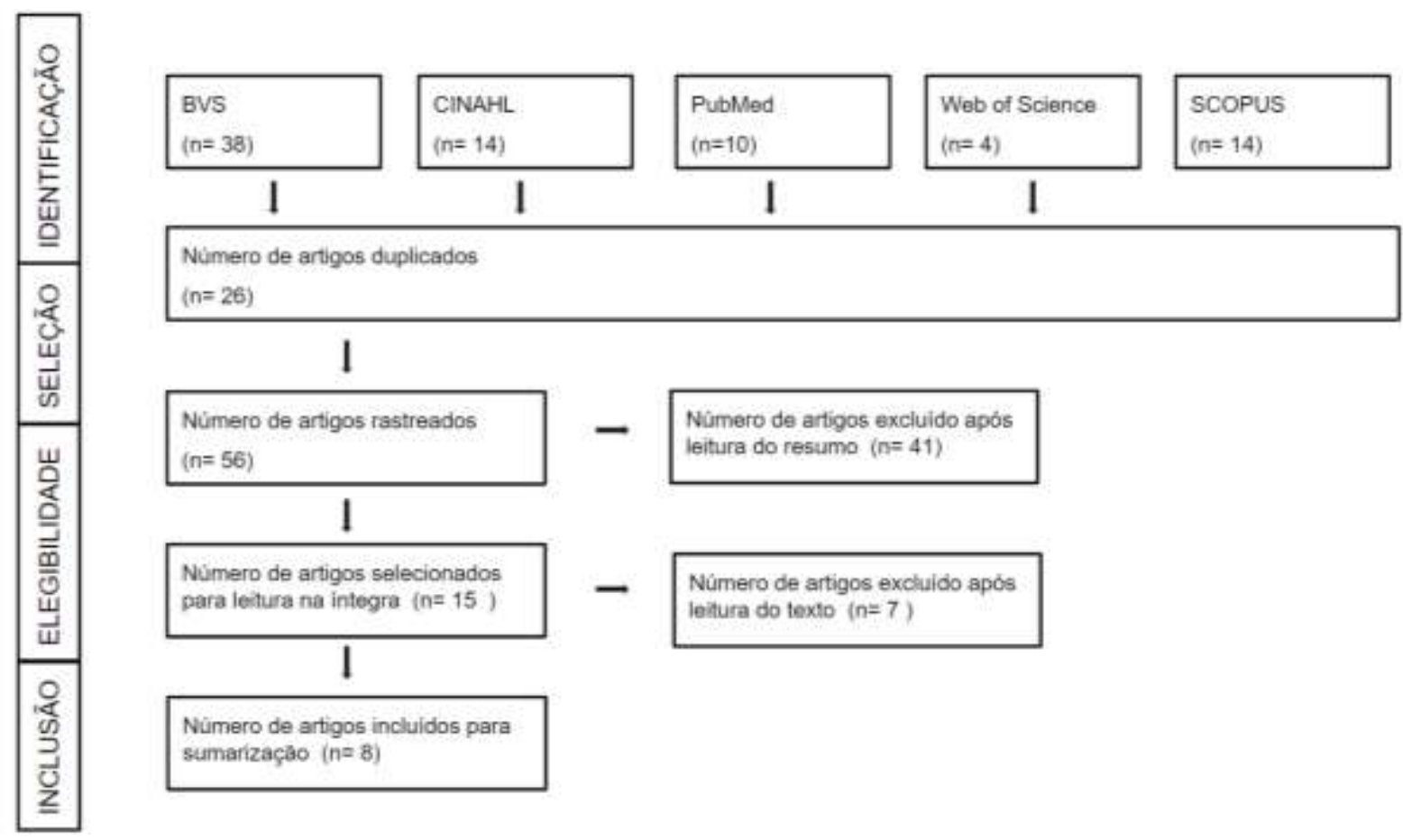

Fonte: Autores (2021).

Após filtragem no programa Start, excluíram-se 26 artigos duplicados ou triplicados e, a partir da leitura do título e resumo e seguindo o critério de gratuidade, totalizaram 15 artigos. Posteriormente foi feita leitura na íntegra, restando 8 artigos no total, pois foram considerados apenas estudos que abordavam alguma tecnologia utilizadas para o ensino e aprendizagem de estudantes de enfermagem durante a pandemia da Covid-19.

A descrição dos autores, ano de publicação, país de origem, tipo de estudo e títulos dos artigos selecionados nesta revisão estão apresentados abaixo (Quadro 2). 
Quadro 2 - Estudos selecionados para a realização da revisão integrativa.

\begin{tabular}{|c|c|c|c|c|}
\hline Estudo & Autor/ano & País & Tipo de estudo & Título \\
\hline E1 & $\begin{array}{l}\text { Malhotra e } \\
\text { Kumar, 2021 }\end{array}$ & $\begin{array}{l}\text { Estados } \\
\text { Unidos }\end{array}$ & $\begin{array}{l}\text { Relato de } \\
\text { experiência }\end{array}$ & $\begin{array}{l}\text { Breaking the COVID-19 Barriers to Health Professional } \\
\text { Team Training With Online Simulation }\end{array}$ \\
\hline $\mathrm{E} 2$ & $\begin{array}{l}\text { Camacho e } \\
\text { Conceição, } 2020\end{array}$ & Brasil & Estudo reflexivo & $\begin{array}{l}\text { Remote teaching in times of the COVID-19 pandemic: } \\
\text { new experiences and challenges }\end{array}$ \\
\hline E3 & Fogg et al, 2020 & $\begin{array}{l}\text { Estados } \\
\text { Unidos }\end{array}$ & Estudo descritivo & $\begin{array}{l}\text { Transitioning from direct care to virtual clinical } \\
\text { experiences during the COVID-19 pandemic }\end{array}$ \\
\hline E4 & Prata et al, 2020 & Brasil & $\begin{array}{l}\text { Relato } \\
\text { experiencia }\end{array}$ & $\begin{array}{l}\text { Mediações pedagógicas de ensino não formal da } \\
\text { enfermagem durante a pandemia de COVID-19 }\end{array}$ \\
\hline E5 & Zhou et al, 2020 & China & Ensaio clínico & $\begin{array}{l}\text { The Distance Teaching Practice of Combined Mode of } \\
\text { Massive Open Online Course Micro-Video for Interns } \\
\text { in Emergency Department During the COVID-19 } \\
\text { Epidemic Period }\end{array}$ \\
\hline E6 & $\begin{array}{l}\text { Bastos et al, } \\
2020\end{array}$ & Brasil & $\begin{array}{l}\text { Relato } \\
\text { experiencia }\end{array}$ & $\begin{array}{l}\text { Ensino remoto emergencial na graduação em } \\
\text { enfermagem: Relato de experiência na covid-19 }\end{array}$ \\
\hline E7 & $\begin{array}{l}\text { Buthelezi e } \\
\text { Wyk, } 2020\end{array}$ & $\begin{array}{l}\text { África do } \\
\text { Sul }\end{array}$ & $\begin{array}{l}\text { Quantitativo } \\
\text { exploratório }\end{array}$ & $\begin{array}{l}\text { The use of an online learning management system by } \\
\text { postgraduate nursing students at a selected higher } \\
\text { educational institution in KwaZulu-Natal, South Africa }\end{array}$ \\
\hline E8 & $\begin{array}{l}\text { Konrad, } \\
\text { Deckers, } 2020\end{array}$ & $\begin{array}{l}\text { Estados } \\
\text { Unidos }\end{array}$ & $\begin{array}{l}\text { Relato } \\
\text { experiência }\end{array}$ & $\begin{array}{l}\text { Nursing fundamentals - supporting clinical competency } \\
\text { online during the COVID-19 pandemic }\end{array}$ \\
\hline
\end{tabular}

Fonte: Autores (2021).

Os artigos incluídos na revisão foram publicados entre o ano de 2020 e 2021, com maior número de publicações no ano de 2020. Destes, três foram desenvolvidos no Brasil, três nos Estados Unidos e os outros na China e África do Sul. Quanto à abordagem metodológica se destaca as pesquisas qualitativas, incluídos quatro relatos de experiências e um estudo reflexivo e descritivo. Restando dois de abordagem quantitativa.

Do conteúdo dos artigos foram extraídos dados referentes ao objetivo dos estudos; tecnologia contemplada e resultados; os quais estão organizados no quadro abaixo (Quadro 3). 
Quadro 3 - Objetivos, Tecnologias Contempladas e Resultados dos estudos selecionados para a realização da revisão integrativa.

\begin{tabular}{|c|c|c|c|}
\hline Estudo & Objetivo & $\begin{array}{l}\text { Tecnologia } \\
\text { contemplada }\end{array}$ & Resultados \\
\hline E1 & $\begin{array}{l}\text { Descrever os detalhes de } \\
\text { workshops de simulação de } \\
\text { emergências obstétricas e } \\
\text { neonatais através de plataforma } \\
\text { de comunicação online. }\end{array}$ & $\begin{array}{l}\text { Zoom e } \\
\text { simulação } \\
\text { clínica virtual }\end{array}$ & $\begin{array}{l}\text { Os workshops de simulação online foram bem } \\
\text { recebidos pelos participantes e as discussões e } \\
\text { comportamentos no formato online foram } \\
\text { semelhantes ao formato presencial, abordando } \\
\text { aspectos como comunicação, trabalho de equipe e } \\
\text { habilidades comportamentais em cenários de } \\
\text { emergências obstétricas. }\end{array}$ \\
\hline E2 & $\begin{array}{l}\text { Refletir sobre o ensino remoto } \\
\text { como oportunidade para novas } \\
\text { experiências e desafios durante a } \\
\text { pandemia da Covid-19. }\end{array}$ & $\begin{array}{l}\text { Google tools: } \\
\text { Meet }\end{array}$ & $\begin{array}{l}\text { A pandemia permitiu uma reflexão sobre as } \\
\text { perspectivas da educação em enfermagem e, no que } \\
\text { se refere ao ensino remoto em plataformas de } \\
\text { comunicação e compartilhamento online, deve-se } \\
\text { propor ações para que seja inclusivo e que garanta } \\
\text { amplo treinamento aos professores para que não } \\
\text { haja barreiras nesse processo. }\end{array}$ \\
\hline E3 & $\begin{array}{l}\text { Desenvolver um protocolo para } \\
\text { uso de simulação em um } \\
\text { ambiente virtual. }\end{array}$ & $\begin{array}{l}\text { Simulação } \\
\text { clínica virtual }\end{array}$ & $\begin{array}{l}\text { A simulação no ambiente virtual se apresenta como } \\
\text { uma metodologia que pode ser empregada para } \\
\text { substituir a aprendizagem clínica durante a } \\
\text { pandemia. As dificuldades evidenciadas nesta } \\
\text { metodologia foram relacionadas a conexões de } \\
\text { internet, falta de motivação para estudar, } \\
\text { dificuldades no ambiente familiar e inexperiência } \\
\text { do corpo docente com um ambiente online. }\end{array}$ \\
\hline $\mathrm{E} 4$ & $\begin{array}{l}\text { Relatar a experiência de } \\
\text { desenvolvimento de mediações } \\
\text { pedagógicas em Ambiente } \\
\text { Virtual de Aprendizagem } \\
\text { (AVA). }\end{array}$ & Moodle & $\begin{array}{l}\text { O curso realizado por meio do AVA foi } \\
\text { considerado eficiente, além de promover a } \\
\text { aprendizagem colaborativa de conhecimentos } \\
\text { acerca da Covid-19, estreitando as relações entre } \\
\text { docentes e educandos. Um dos fatores limitantes } \\
\text { que impossibilitou o acesso dos estudantes ao curso } \\
\text { foi a conexão com a internet. }\end{array}$ \\
\hline E5 & $\begin{array}{l}\text { Observar e analisar o efeito da } \\
\text { aplicação da modalidade } \\
\text { combinada do MOOC na prática } \\
\text { de ensino a distância de internos } \\
\text { de pronto-socorro. }\end{array}$ & MOOC & $\begin{array}{l}\text { O MOOC é uma alternativa para quando os cursos } \\
\text { presenciais e práticos não podem mais ser } \\
\text { realizados. A utilização de vídeos de treinamento, } \\
\text { possibilita que os estudantes transformem a } \\
\text { educação passiva em aprendizagem ativa, } \\
\text { combinando melhor a teoria com a prática. }\end{array}$ \\
\hline E6 & $\begin{array}{l}\text { Descrever a experiência no } \\
\text { ensino remoto emergencial para } \\
\text { as aulas teóricas. }\end{array}$ & $\begin{array}{l}\text { Big } \quad \text { Blue } \\
\text { Button; } \\
\text { Microsoft } \\
\text { Teams } \\
\text { Webex. }\end{array}$ & $\begin{array}{l}\text { Aulas com metodologias não dinâmicas e pouco } \\
\text { criativas não favorecem a participação dos } \\
\text { discentes. A experiência favoreceu a aquisição de } \\
\text { conhecimentos sobre as plataformas virtuais e a } \\
\text { implantação de aulas e treinamento dos docentes, } \\
\text { bem como favoreceu a aproximação entre } \\
\text { educador-educando. }\end{array}$ \\
\hline
\end{tabular}




\begin{tabular}{|c|c|c|c|}
\hline E7 & $\begin{array}{l}\text { Explorar as percepções dos } \\
\text { alunos sobre e-learning, seus } \\
\text { desafios percebidos com a } \\
\text { tecnologia em um módulo } \\
\text { obrigatório de pós-graduação } \\
\text { em enfermagem, bem como as } \\
\text { associações entre rados } \\
\text { demográficos } \\
\text { listados. }\end{array}$ & Moodle & $\begin{array}{l}\text { A coorte expressou opiniões positivas sobre o uso } \\
\text { da tecnologia, } 70 \% \text { relataram a primeira exposição } \\
\text { ao Moodle da universidade e } 68,3 \% \text { tiveram acesso } \\
\text { a um computador. A maioria }(66,7 \%) \text { expressou ter } \\
\text { habilidades limitadas em TIC e dificuldade para } \\
\text { usar o Moodle. Foram encontradas associações } \\
\text { estatisticamente significativas entre a habilidade de } \\
\text { usar o Moodle e proficiência em inglês, } \\
\text { conhecimento de informática, disponibilidade de } \\
\text { suporte técnico e acesso a computadores. }\end{array}$ \\
\hline E8 & $\begin{array}{l}\text { Discutir as etapas percorridas } \\
\text { para assegurar que os objetivos } \\
\text { de aprendizagem do curso } \\
\text { clínico de enfermagem } \\
\text { fundamental foram atingidos. }\end{array}$ & $\begin{array}{l}\text { Zoom, } \\
\text { Simulações } \\
\text { realidade } \\
\text { virtual } \\
\text { realidade } \\
\text { aumentada }\end{array}$ & $\begin{array}{l}\text { As escolas de enfermagem precisam oferecer } \\
\text { treinamento formal sobre as melhores práticas em } \\
\text { educação online para garantir que o instrutor esteja } \\
\text { equipado com o conhecimento necessário para } \\
\text { ministrar com sucesso um curso online. A } \\
\text { implementação de técnicas de aprendizado online } \\
\text { como um complemento regular da prática clínica } \\
\text { tradicional pode melhorar os resultados do } \\
\text { aprendizado e transformar os alunos em graduados } \\
\text { prontos para trabalhar com segurança. }\end{array}$ \\
\hline
\end{tabular}

Fonte: Autores (2021).

Como observado acima, os estudos contemplaram intervenções educacionais remotas como simulação clínica em ambiente virtual, uso de plataformas de ensino e de diversas TICs. As plataformas de ensino encontradas foram o Modular Object-Oriented Dynamic Learning Environment (Moodle) e o Massive Open Online Course (MOOC). Já as entre as TICs adotadas, destacam-se as plataformas de comunicação online como Zoom, Google Meet, Big Blue Button; Microsoft Teams e Webex.

\section{Discussão}

A pandemia da Covid-19 aconteceu de forma inesperada e os impactos causados pelas medidas de isolamento e distanciamento social impuseram um desafio para diversos setores da sociedade, destacando-se o educacional (Konrad, Fitzgerald \& Deckers, 2021). Com a suspensão das aulas presenciais nas instituições de ensino superior, estudantes e professores migraram para atividades online, por meio de plataformas de comunicação como: Skype, Google Hangout e Zoom, bem como plataformas de aprendizagem: Moodle, Microsoft Teams, Google Classroom, entre outros. Essas plataformas permitem aos usuários realizar chamadas de vídeos e áudio para diversos fins, desde reuniões de trabalho até aulas online (Moreira, Henriques \& Barros, 2020), como identificado nesta revisão, onde quatro estudos (E1, E2, E6 e E8) apresentam o uso das plataformas de comunicação.

O advento de tecnologias ao ensino é um processo que tem se acelerado naturalmente, no ritmo dos avanços da ciência e tecnologia. No entanto, a crise sanitária causada pela pandemia da Covid-19 exerceu um papel catalisador para o uso das TED e TIC na educação (Lira et al, 2020). O estudo reflexivo de Camacho (2020), acerca do ensino remoto da enfermagem durante pandemia do novo coronavírus, aponta que algumas ações são necessárias para garantir que o ensino através de plataformas de comunicação e compartilhamento online seja mais inclusiva, a começar pelo amplo treinamento de professores para o uso dessas tecnologias, tais como Google Tools.

Nesse sentido, o relatório sobre a implantação do ERE em uma faculdade do sudeste do Brasil revelou que a plataforma 
Google Classroom foi a mais utilizada para as aulas do curso de Medicina, sendo necessária a capacitação docente, adaptação dos estudantes e adoção de estratégias financeiras, como bolsa-auxílio trabalho, para garantir o acesso de todos os estudantes (Appenzeler, Menezes, Santos, Padilha, Graça \& Bragança, 2020).

Em um estudo realizado nos Estados Unidos, evidencia-se que a utilização da plataforma Zoom e da simulação de realidade virtual e realidade aumentada são satisfatórias, tanto para estudantes quanto para professores, pois a aula online pode passar a ser um complemento regular do ensino tradicional e melhorar os resultados do aprendizado, a fim de formar os alunos para uma prática mais segura. No entanto, uma vez que a maioria dos docentes ensinam como foram ensinados, houve dificuldade para adaptar as metodologias de ensino ao formato online, sendo necessário um treinamento específico para tal (Konrad et al, 2020).

Corroborando com essa premissa, um estudo realizado no nordeste do Brasil que descreveu a experiência no ERE para a graduação em Enfermagem durante a pandemia da Covid-19, apontou algumas dificuldades e desafios por parte de docentes e discentes com o mundo virtual. Foi mencionada a necessidade de criatividade dos professores para que os estudantes participassem das aulas, bem como atenção aos horários, dedicação e adequação do ambiente familiar. Nesse sentido, esse tipo de formato pode gerar maior carga de trabalho pela necessidade de aprender a reconduzir a sala de aula e por requerer diversidade das atividades. Ademais, alguns alunos podem se sentir sobrecarregados com a dinâmica do ER em virtude do cenário pandêmico ter modificado as suas rotinas de vida, como, por exemplo, a necessidade de trabalhar nesse período (Bastos, Canavarro, Campos, Schulz, Santos \& Santos, 2020).

Assim como as demais estratégias, o uso das plataformas de comunicação para o ensino enfrenta como desafio problemas de conexão com a internet, baixa adesão dos alunos em adotar o ensino online e capacidade dos professores em dominar as ferramentas virtuais. Portanto, é necessário que o aluno participe ativamente, construindo o seu próprio processo de aprendizagem, com auxílio do professor para oferecer feedback em relação ao seu desenvolvimento (Jowsey, Foster, CooperIoelu \& Jacobs, 2020).

No ensino da enfermagem, é comum realizar práticas em laboratórios de simulação para aperfeiçoamento do conhecimento e aquisição de habilidades para procedimentos. As tecnologias possibilitam elaborar ambientes virtuais de simulação que se assemelham às aulas de laboratório. (Fonseca, Aredes, Fernandes, Batalha, Apóstolo, Martins \& Rodrigues, 2016). Com a pandemia da Covid-19, devido às restrições de distanciamento, estes ambientes foram uma alternativa para substituir as práticas presenciais, garantindo a segurança dos estudantes e a continuidade da aprendizagem (Fogg, Wilson, Trinka, Campbell, Thomson, Merritt, Tietze \& Prior, 2020; Malhotra \& Kumar, 2021).

Nesta revisão foram encontrados em três estudos (E1, E3, E8) o uso da simulação em ambiente virtual durante a pandemia da Covid-19. Um estudo relata a implantação da prática online por uma Universidade dos Estados Unidos, na qual fizeram uso de programas de simulação clínica, que replicam experiências do ambiente hospitalar proporcionando aos alunos, através do meio eletrônico, o desenvolvimento de competências para avaliação e tomada de decisão em relação aos cuidados de enfermagem com o paciente. Um dos obstáculos encontrados no uso dessa metodologia foi o acesso à internet $\mathrm{e}$ dificuldades do corpo docente em manusear as tecnologias virtuais (Fogg et al, 2020).

Outra experiência de simulação online foi desenvolvida na Austrália, em que se realizavam workshops online de simulação de emergências no momento do parto. Houve possibilidade de participação de estudantes e profissionais de saúde de maneira interprofissional: médicos, enfermeiras e enfermeiras obstetras. Essa modalidade de simulação foi bem recebida pelos participantes, de modo que foi observado que as discussões e comportamentos dos alunos nesse formato foram semelhantes ao presencial, abordando aspectos como comunicação, trabalho de equipe e habilidades comportamentais em cenários de emergências obstétricas (Malhotra \& Kumar, 2021). 
Um estudo que avaliou a experiência de alunos que passaram por um exercício de descontaminação através do ambiente virtual de simulação, obteve um feedback positivo, demonstrando que este é um método de aprendizagem que permite aos alunos praticar o conteúdo aprendido em um ambiente seguro e realista, além de que os alunos apresentaram-se entusiasmado com a metodologia (Ulrich, Farra, Smith \& Hodgson, 2014). As principais implicações no uso de simulação estão relacionadas ao treinamento dos professores para o uso das ferramentas, organização do cenário e conteúdo de aprendizagem, além do tempo e recursos financeiros necessários para o emprego desta tecnologia (Oliveira, Prado \& Kempfer, 2014).

Levando em consideração a pandemia da Covid-19, que impede a presença dos alunos em laboratórios, a simulação no ambiente online pode ser um substituto eficaz quando executada corretamente. Apesar dos obstáculos como o acesso a internet, esta estratégia tem potencial para garantir aos alunos o desenvolvimento de competências profissionais durante a crise sanitária (Fogg et al, 2020; Malhotra \& Kumar, 2021).

As Plataformas de ensino são sistemas desenvolvidos para gestão da aprendizagem, com intuito de otimizar a aprendizagem e oferecer ferramentas para o desenvolvimento autônomo dos estudantes. Estas proporcionam um conjunto de funcionalidades que permitem a criação de cursos, aplicação de aulas, produção de conteúdo, compartilhamento de informações e interação entre professor e aluno, além de permitir a monitorização, registro e avaliação (Gabardo, Quevedo \& Ulbricht, 2010). As plataformas de ensino encontradas nesta revisão foram o Moodle (E4 e E7) e MOOC (E5).

O MOOC é uma modalidade de curso oferecido por meio virtual, geralmente por instituições acadêmicas que podem ser acessados em qualquer local e horário. É realizado por módulos e avalia o aprendizado do aluno conforme a progressão no curso, é um método de aprendizagem flexível e de fácil acesso (Teixeira, Miranda, Oliveira \& Pinto, 2018).

Um estudo foi realizado na China acerca do uso da aplicação da tecnologia MOOC durante o período pandêmico da Covid-19 na prática do ensino a distância de estagiários de um pronto-socorro. Segundo os resultados encontrados, em comparação com os métodos tradicionais de ensino, o efeito do modo combinado do micro vídeo MOOC na prática de enfermagem de emergência é o mesmo dos métodos tradicionais de ensino. Entretanto, as evidências apontam para uma satisfação maior, por isso é mais adequado para ser usado na prática de enfermagem durante a crise sanitária, de forma a reduzir efetivamente a infecção cruzada entre médicos, enfermeiras e corpo docente (Zhou, Huang, Cheng \& Xiao, 2020).

Em contrapartida, outro estudo que analisou a avaliação de 118 pessoas, entre elas enfermeiras $(61,9 \%)$ e estudantes de graduação em Enfermagem (38,1\%) sobre um MOOC desenvolvido por uma Universidade do sul do Brasil demonstrou que esta modalidade obteve uma alta taxa de evasão. No entanto, o curso realizado pela instituição atendeu as expectativas de 94,9\% dos participantes, sendo que esta estratégia colabora efetivamente com a educação permanente dos profissionais da área da saúde. O desafio encontrado para a utilização do MOOC foi o domínio da informática, sendo necessário o treinamento dos usuários para aplicá-lo (Parulla, Cogo \& Dal Pai, 2020).

O Moodle, também conhecido como Ambiente Virtual de Aprendizagem (AVA), é uma plataforma de e-learning (ensino eletrônico que corresponde a um modelo não presencial apoiado em TICs), utilizada por alunos e professores como ferramenta de apoio ao ensino a distância. Nela há fóruns de discussão e salas de bate-papo para aprofundamento da temática e esclarecimento de dúvidas. Os professores podem ainda publicar textos-base e propor questões para reflexão e fixação do conteúdo, bem como determinar tarefas e disponibilizar os arquivos para download (Instituto Federal do Pará, 2019).

Com o objetivo de explorar as percepções dos alunos sobre e-learning e os desafios percebidos com a tecnologia em um curso de pós-graduação em enfermagem, um estudo foi realizado na África do Sul. A maioria dos participantes (66,7\%) expressou ter habilidades limitadas em TICs e dificuldade para usar o Moodle. Foram encontradas associações estatisticamente significativas entre a habilidade de usar o Moodle e proficiência em inglês, conhecimento de informática, disponibilidade de suporte técnico e acesso a computadores (Buthelezi \& Van Wyk, 2020). Em outro estudo no qual relataram as experiências do 
uso do AVA em uma faculdade de enfermagem do Sudeste do Brasil, revela que o Moodle é uma estratégia eficiente para ser aplicado durante a pandemia da Covid-19, possibilita a aprendizagem e estreita as relações entre professor e aluno (Prata, Mello, Costa e Silva \& Faria, 2020).

No que diz respeito às avaliações realizadas remotamente, um estudo realizado em 2020 em uma Universidade da Jordânia objetivou avaliar a experiência de alunos de diversos cursos da área da saúde em relação à percepção desses sobre as avaliações/exames realizados remotamente por intermédio de ferramentas eletrônicas. Apenas um terço dos alunos participantes da pesquisa preferiu esta modalidade quando comparada com a presencial. A desonestidade e má conduta no exame apareceu como um dos maiores desafios, entretanto, como sugestão para redução desse comportamento foi mencionada a substituição do exame por outras formas como: a utilização de diferentes formulários de avaliação, a utilização de soluçães de supervisão online e a consideração de notas obrigatórias de aprovação/reprovação (Elsalem, Al-Azzam, Jum'ah \& Obeidat, 2021).

As limitações deste estudo foram relacionadas ao baixo número de publicações de acesso livre relacionadas à temática durante a pandemia da Covid-19, o que não permitiu uma análise ampla sobre as tecnologias utilizadas para o ensino da Enfermagem neste cenário atípico.

\section{Considerações Finais}

Com a pandemia da Covid-19, verificou-se a necessidade da implementação de novas metodologias para continuidade do aprendizado. Neste estudo, identificamos o uso de diferentes tecnologias para o ensino da enfermagem, destacando-se a simulação clínica em ambiente virtual, plataformas digitais de ensino e plataformas de comunicação online.

O uso destas tecnologias demonstrou eficiência em substituir os modelos tradicionais de aprendizagem durante a crise sanitária causada pelo novo coronavírus, entretanto evidenciaram-se dificuldades na incorporação destas ferramentas por alunos e professores, principalmente relacionadas a problemas de infraestrutura e capacitação tecnológica dos educadores. Há também a necessidade de explorar mais estes recursos em busca de proporcionar um ambiente adequado para aprendizagem ativa e desenvolvimento autônomo do estudante em busca do conhecimento. Espera-se que este estudo incentive instituições de ensino, professores e alunos a ampliar a utilização e descobrir potencialidades destes recursos tecnológicos no processo de ensino-aprendizagem.

\section{Referências}

Andrade, M. A. (2019). O uso das TICs na educação à distância. (Monografia). Instituto Federal Goiano.

Appenzeller, S., Menezes, F. H., Santos, G. G., Padilha, R. F., Graça, H. S., \& Bragança, F. J. (2020). Novos Tempos, Novos Desafios: Estratégias para Equidade de Acesso ao Ensino Remoto Emergencial. Rev. bras. educ. med., 44 (1).

Bastos, M. D. C., Canavarro, D. D. A., Campos, L. M., Schulz, R. D. S., Santos, J. B. D., \& Santos, C. F. D. (2020). Ensino remoto emergencial na graduação em enfermagem: relato de experiência na covid-19. Revista Mineira de Enfermagem, 24, 1-6.

Brasil. Ministério da Educação. (2019). Portaria N²117 de 06 de Dezembro de 2019. Dispõe sobre a oferta de carga horária na modalidade de Ensino a Distância - EaD em cursos de graduação presenciais ofertados por Instituições de Educação Superior pertencentes ao Sistema Federal de Ensino. Edição 239. Seção 1. Página 131. Brasília.

Brasil, Ministério da Educação (2020a). Portaria No 343, de 17 de Março de 2020. Dispõe sobre a substituição das aulas presenciais por aulas em meios digitais enquanto durar a situação de pandemia do Novo Coronavírus. Edição: 53, Seção: 1, Pag. 39.

Brasil. Ministério da Educação (2020b). Portaria n ${ }^{\circ} 544$, de 16 de junho de 2020. Dispõe sobre a substituição das aulas presenciais por aulas em meios digitais, enquanto durar a situação de pandemia do novo coronavírus, e revoga as Portarias MEC $\mathrm{n}^{\circ} 343$, de 17 de março de 2020 , $\mathrm{n}^{\circ} 345$, de 19 de março de 2020 , e $\mathrm{n}^{\circ}$ 473, de 12 de maio de 2020. Diário Oficial da União, Brasília, DF, 2020. p.62.

Buthelezi, L. I., \& Van Wyk, J. M. (2020). The use of an online learning management system by postgraduate nursing students at a selected higher educational institution in KwaZulu-Natal, South Africa. African Journal of Health Professions Education, 12(4), 211-214. 
Camacho, A. C. L. F. (2020). Remote teaching in times of the COVID-19 pandemic: new experiences and challenges. Online Brazilian Journal of Nursing, 19(4).

Conselho Federal de Enfermagem (2020). Por que o Cofen é contra o EaD em Enfermagem? http://biblioteca.cofen.gov.br/videos/por-que-o-cofen-e-contra-oead-em-enfermagem/.

Elsalem, L., Al-Azzam, N., Jum'ah, A. A., \& Obeidat, N. (2021). Remote E-exams during Covid-19 pandemic: A cross-sectional study of students' preferences and academic dishonesty in faculties of medical sciences. Annals of Medicine and Surgery, 62, 326-333.

Góes, F. D. S. N., de Camargo, R. A. A., Hara, C. Y. N., \& Fonseca, L. M. M. (2014). Tecnologias educacionais digitais para educação profissional de nível médio em enfermagem. Revista Eletrônica de Enfermagem, 16(2), 453-61.

Godoi, M., Beraldo Kawashima, L., Gomes, L., \& Caneva, C. (2020). O ensino remoto durante a pandemia de covid-19: desafios, aprendizagens e expectativas dos professores universitários de Educação Física. Research, Society and Development, 9(10), 1-19.

Fogg, N., Wilson, C., Trinka, M., Campbell, R., Thomson, A., Merritt, L., Tietze, M., \& Prior, M. (2020). Transitioning from direct care to virtual clinical experiences during the COVID-19 pandemic. Journal of Professional Nursing, 36(6), 685-691.

Fonseca, L. M. M., Aredes, N. D. A., Fernandes, A. M., Batalha, L. M. D. C., Apóstolo, J. M. A., Martins, J. C. A., \& Rodrigues, M. A. (2016). Simulação por computador e em laboratório no ensino em enfermagem neonatal: as inovações e o impacto na aprendizagem. Revista Latino-Americana de Enfermagem, 24.

Gabardo, P., de Quevedo, S. R., \& Ulbricht, V. R. (2010). Estudo comparativo das plataformas de ensino-aprendizagem. Encontros Bibli: revista eletrônica de biblioteconomia e ciência da informação, (2. sem.), 65-84.

Instituto Federal do Pará. (2019). Afinal, o que é Moodle? https://ctead.ifpa.edu.br/noticias/544-afinal-o-que-e-moodle

Jowsey, T., Foster, G., Cooper-Ioelu, P., \& Jacobs, S. (2020). Blended learning via distance in pre-registration nursing education: A scoping review. Nurse education in practice, $44,102775$.

Konrad, S., Fitzgerald, A., \& Deckers, C. (2021). Nursing fundamentals-supporting clinical competency online during the COVID-19 pandemic. Teaching and Learning in Nursing, 16(1), 53-56.

Lahti, M., Hätönen, H., \& Välimäki, M. (2014). Impact of e-learning on nurses' and student nurses knowledge, skills, and satisfaction: a systematic review and meta-analysis. International journal of nursing studies, 51(1), 136-149.

Lira, A. L. B. D. C., Adamy, E. K., Teixeira, E., \& Silva, F. V. D. (2020). Educação em enfermagem: desafios e perspectivas em tempos da pandemia COVID19. Revista Brasileira de Enfermagem, 73(2).

Malhotra, A., \& Kumar, A. (2021). Breaking the COVID-19 Barriers to Health Professional Team Training With Online Simulation. Simulation in Healthcare, $16(1), 80$.

Moher, D., Liberati, A., Tetzlaff, J., \& Altman, D. G. (2010). Preferred reporting items for systematic reviews and meta-analyses: the PRISMA statement. Int J Surg, 8(5), 336-341.

Mendes, K. D. S., Silveira, R. C. D. C. P., \& Galvão, C. M. (2008). Revisão integrativa: método de pesquisa para a incorporação de evidências na saúde e na enfermagem. Texto \& contexto-enfermagem, 17(4), 758-764.

Moreira, J. A., Henriques, S., \& Barros, D. M. V. (2020). Transitando de um ensino remoto emergencial para uma educação digital em rede, em tempos de pandemia. Dialogia, 34, 351-364.

Oliveira, C. (2015). TIC'S na educação: a utilização das tecnologias da informação e comunicação na aprendizagem do aluno. Pedagogia em ação, 7(1).

Oliveira, S. N. D., Prado, M. L. D., \& Kempfer, S. S. (2014). Utilização da simulação no ensino da enfermagem: revisão integrativa. Revista Mineira de Enfermagem, 18(2), 487-504.

Paese, C. R. (2012). Educação a distância (ead) e o uso das tecnologias de informação e comunicação (tics), baseada em ambientes virtuais de aprendizagem (ava) Algumas Reflexões sobre a Importância da Tutoria On-Line. Itinerarius Reflectionis, 8(1).

Parulla, C. D., Cogo, A. L. P., \& Dal Pai, D. (2020). Avaliação de estudantes de Enfermagem e enfermeiros de um curso online, aberto e massivo (MOOC). Research, Society and Development, 9(9).

Peters, M. D., Marnie, C., Tricco, A. C., Pollock, D., Munn, Z., Alexander, L., \& Khalil, H. (2020). Updated methodological guidance for the conduct of scoping reviews. JBI Evidence Synthesis, 18(10), 2119-2126.

Prata, J. A., Mello, A. S. D., Costa e Silva, F. V., \& Faria, M. G. D. A. (2020). Mediações pedagógicas de ensino não formal da enfermagem durante a pandemia de COVID-19. Revista Brasileira de Enfermagem, 73(2).

Saldanha, L. C. D. (2020). O discurso do ensino remoto durante a pandemia de COVID-19. Revista Educação e Cultura Contemporânea, 17(50), 124-144.

Silveira, M. D. S., \& Cogo, A. L. P. (2017). Contribuições das tecnologias educacionais digitais no ensino de habilidades de enfermagem: revisão integrativa. Revista Gaúcha de Enfermagem, 38(2).

Teixeira, E., Fernandes, J. D., Andrade, A. D. C., Silva, K. L., Rocha, M. E. M. O. D., \& Lima, R. J. D. O. (2013). Panorama dos cursos de Graduação em Enfermagem no Brasil na década das Diretrizes Curriculares Nacionais. Revista Brasileira de Enfermagem, 66, 102-110. 
Research, Society and Development, v. 10, n. 6, e13610615576, 2021

(CC BY 4.0) | ISSN 2525-3409 | DOI: http://dx.doi.org/10.33448/rsd-v10i6.15576

Teixeira, A., Miranda, B., Oliveira, I., \& Pinto, M. D. C. T. (2018). MOOC “Competências digitais para professores”: uma prática formativa inovadora. RIED. Revista Iberoamericana de Educación a Distancia, 243-261.

Ulrich, D., Farra, S., Smith, S., \& Hodgson, E. (2014). The student experience using virtual reality simulation to teach decontamination. Clinical Simulation in Nursing, 10(11), 546-553.

World Health Organization. (2020). WHO announces COVID-19 outbreak a pandemic. http://www.euro.who.int/en/health-topics/healthemergencies/coronavirus-covid-19/news/news/2020/3/who-announces-covid-19-outbreak-a-pandemic.

Zhou, T., Huang, S., Cheng, J., \& Xiao, Y. (2020). The distance teaching practice of combined mode of massive open online course micro-video for interns in emergency department during the COVID-19 epidemic period. Telemedicine and e-Health, 26(5), 584-588. 